\title{
A DSP-BASED BEAM CURRENT MONITORING SYSTEM FOR MACHINE PROTECTION USING ADAPTIVE FILTERING*
}

\author{
J. Musson ${ }^{\dagger}$, H. Dong, R. Flood, C. Hovater \\ Thomas Jefferson National Accelerator Facility, Newport News, VA \\ J. Hereford, Christopher Newport University, Newport News, VA 23601
}

\begin{abstract}
The CEBAF accelerator at Jefferson Lab is currently using an analog beam current monitoring (BCM) system for its machine protection system (MPS), which has a loss accuracy of $2 \mu \mathrm{A}$. Recent burn-through simulations predict catastrophic beam line component failures below 1 $\mu \mathrm{A}$ of loss, resulting in a blind spot for the MPS. Revised MPS requirements target an ultimate beam loss accuracy of $250 \mathrm{nA}$. A new beam current monitoring system has been developed which utilizes modern digital receiver technology and digital signal processing concepts. The receiver employs a direct-digital down converter integrated circuit, mated with a Jefferson Lab digital signal processor VME card. Adaptive filtering is used to take advantage of current-dependent burn through rates. Benefits of such a system include elimination of DC offsets, generic algorithm development, extensive filter options, and interfaces to UNIX -based control systems.
\end{abstract}

\section{BEAM CURRENT MONITOR}

The current BCM system uses a front-end converter to produce a $1 \mathrm{MHz}$ intermediate frequency (IF) from the $1.497 \mathrm{GHz}$ beam cavity signal. The IF signal is envelopedetected using a true RMS-DC converter, where the output is compared to the other end-station signals and injector signal. Resulting beam loss is integrated, and a fast-shutdown occurs if the integrated loss exceeds a threshold of $5000 \mu \mathrm{A}-\mu \mathrm{S}[1]$.

Due to the nature of the DC detection and summation circuits, the resolution cannot be made better than $2 \mu \mathrm{A}$, which is not sufficient for complete machine protection.

\section{DIGITAL DOWNCONVERTER (DDC)}

In an attempt to overcome limitations posed by analog electronics and non-ideal RF circuit elements, a digital scheme was proposed. The $1 \mathrm{MHz}$ IF is oversampled by a 14-bit A/D running at 50 MSPS. Oversampling provides the benefit of reducing the quantization noise of the $A / D$ converter by spreading the noise spectrum out to $25 \mathrm{MHz}$. The digitized signal is sent to a DDC, where the signal is heterodyned and decomposed to in-phase and quadrature (I \& Q) components through the use of a numerically controlled oscillator (NCO) and quadrature multiplier.

Subsequent narrowband Finite-Input-Response (FIR) filtering is required for a number of reasons: (i) to remove any residual aliasing components due to downconversion and decimation, (ii) to remove higher-order components

*Work supported by the U.S. Department of Energy, contract DEAC05-84ER40150

${ }^{\dagger}$ musson@jlab.org from the IF-to-baseband heterodyne conversion, and (iii) to decrease quantization noise[2].

\subsection{DDC Design}

The primary design constraints for the DDC were dictated by the response specifications of the MPS/BCM system, namely: (1) $100 \mathrm{kHz} \mathrm{BW,} \mathrm{(2)} 50 \mathrm{MHz}$ input sample rate, (3) a maximum output sample rate of 2 MSPS to provide at least a $250 \mathrm{kHz}$ output signal bandwidth and (4) a maximum latency of $20 \mu \mathrm{s}$. In all, a $150 \mu \mathrm{s}$ FSD is required for the $5000 \mu \mathrm{A}-\mu$ s charge threshold. The remainder of the FSD time budget is left for digital signal processing $(100 \mu \mathrm{s})$, measurement system delay $(20 \mu \mathrm{s})$, and time to empty the accelerator $(30 \mu \mathrm{s})$. Matlab[3] was used extensively in the following calculations and simulations, while most FIR design and results were performed using System View[4].

The following notation will be used :

$$
\begin{aligned}
& \mathrm{Q}=\text { number of FIR filter taps } \\
& \mathrm{R}=\text { decimation prior to FIR filter stage } \\
& \tau=\text { latency }(\mu \mathrm{s}) \\
& \mathrm{F}_{\text {samp }}=\text { input sample rate of the FIR filter }
\end{aligned}
$$

The latency of the FIR filter is determined by:

$$
\tau=\mathrm{Q} / \mathrm{F}_{\text {samp }}=(\mathrm{QR}) / 50 \mathrm{MHz}
$$

A Q vs R plot with a latency of $20 \mu$ s superimposed is shown in Figure 1.

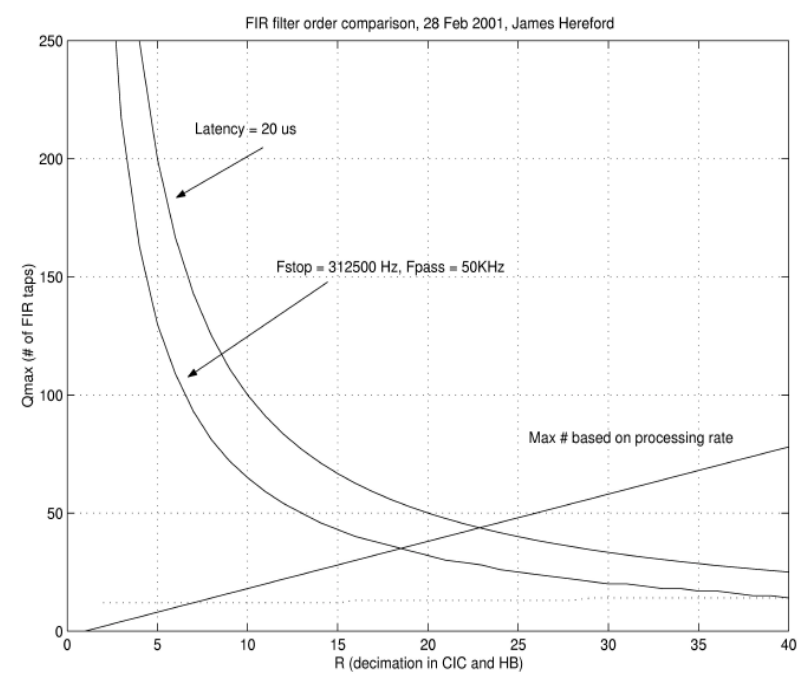

Figure 1: Decimation, R, vs number of FIR taps (Q). 20 $\mu$ s latency contour added for reference. 
An additional FIR requirement, specific to the Intersil HSP50214B DDC, is that the frequency of the FIR clock must exceed a minimum value to ensure completing a filter calculation before the result is required for output. This requirement[5] can be approximated by:

$$
\mathrm{Q} \approx 2(\mathrm{R}-1)
$$

and appears as a straight line on the $\mathrm{Q}$ vs $\mathrm{R}$ plot (see Figure 1).

The resulting latency and filter performance are plotted as Fstop vs $R$, and shown in Figure 2, where $F_{\text {stop }}$ is the minimum stopband frequency of the FIR lowpass filter.

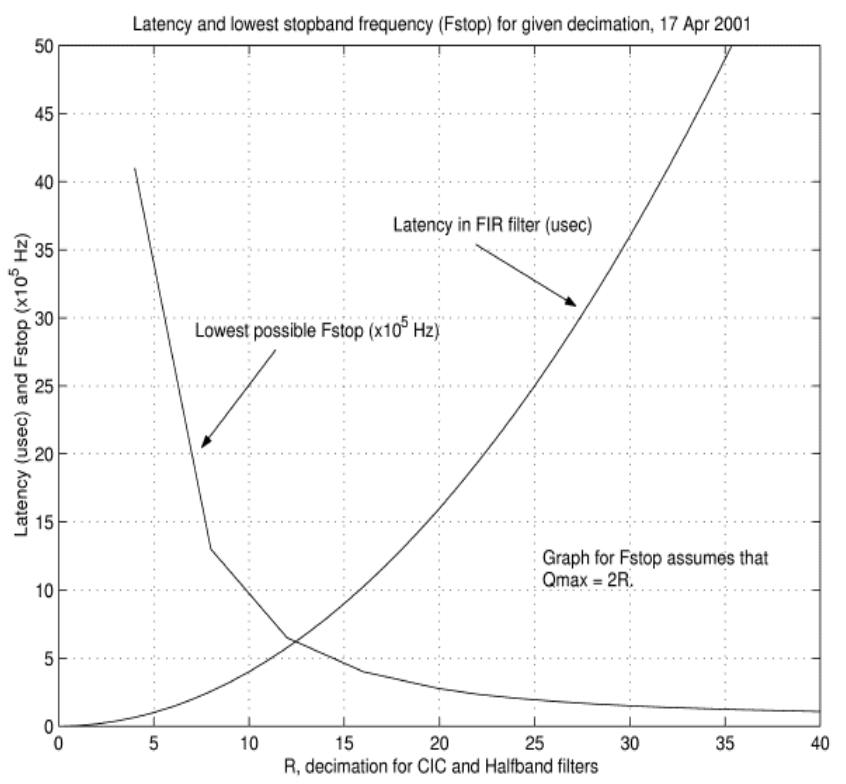

Figure 2: Decimation, R, vs Latency and stopbandwidth, $\mathrm{F}_{\text {stop. }}$.

The minimum value of $\mathrm{R}$ was calculated to be greater than 25 , such that the output sample rate would be less than 2 MSPS. R should be a multiple of 2 to facilitate distribution between an onboard Cascaded Integrator Comb (CIC) and halfband filter. A value of $\mathrm{R}=28$ provided the most flexibility in implementation, as several factoring combinations are possible. The resulting FIR filter produced a filter having a latency of $20 \mu \mathrm{s}$, and $98 \mathrm{kHz} 3 \mathrm{~dB}$ low-pass bandwidth. The final design values are summarized in Table 1.

Table 1: FIR Filter Specifications

\begin{tabular}{|l|l|}
\hline Specification & Value \\
\hline Decimation $\mathrm{R}$ & 28 \\
\hline Passband Frequency $\mathrm{F}_{\text {pass }}$ & $50 \mathrm{kHz}$ \\
\hline Passband Attenuation $\mathrm{A}_{\text {pass }}$ & $0.1 \mathrm{~dB}$ \\
\hline Number of filter taps $\mathrm{Q}$ & 36 \\
\hline Stopband attenuation $\mathrm{A}_{\text {stop }}$ & $70 \mathrm{~dB}$ \\
\hline Input sample rate & $50 \mathrm{MHz} / 28=1.79 \mathrm{MHz}$ \\
\hline Stopband frequency $\mathrm{F}_{\text {stop }}$ & $196 \mathrm{kHz}$ \\
\hline
\end{tabular}

\section{DIGITAL SIGNAL PROCESSOR (DSP)}

In addition to filtering provided by the DDC, a Texas Instruments TSM320C6711 DSP is employed to provide a means of filtering low $\mathrm{S} / \mathrm{N}$ input signals, by varying the filter parameters in real-time to achieve a specific criteria (ie maximize $\mathrm{S} / \mathrm{N}$ ). This process is known as adaptive filtering or equalization.

\subsection{Least-Mean Squares (LMS) Algorithm}

Adaptive filters have recently seen use in controls to filter out narrowband noise, as well as remove discrete sinusoid components. The most prevalent use of such filters is in the area of active noise cancellation within radio headsets and aircraft. These applications try to suppress the sinusoidal or coherent noise (such as from motors), while passing the incoherent noise. This BCM application attempts to preserve the sinusoidal tone, produced by intentionally mistuning the DDC NCO by 1 $\mathrm{kHz}$. The schematic is commonly referred to an adaptive predictor, and is shown in Figure 3a.
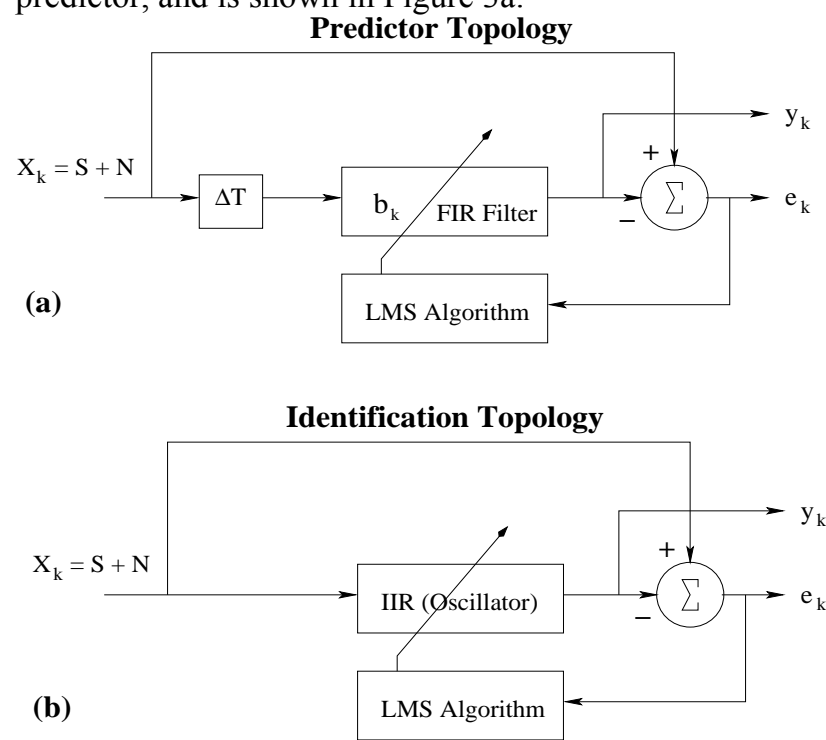

Figure 3: Adaptive prediction topology is shown at (a). $\mathrm{X}_{\mathrm{k}}$ is input signal plus noise, while predicted and error signals are $Y_{k}$ and $e_{k}$, respectively. FIR coefficients are denoted by $b_{k}$. Adaptive Identification is shown in (b).

FIR filter coefficients are found by the LMS algorithm which seeks to reduce the mean quadratic error, $\mathrm{e}_{\mathrm{k}}{ }^{2}$, resulting from the difference between the actual signal and the best estimate of the signal. In theory, the $e_{k}$ contains only the stochastic signal contribution, while the filtered component, $\mathrm{y}_{\mathrm{k}}$, is the deterministic component, or in this case, the $1 \mathrm{kHz}$ tone:

Let:

$$
\mathrm{C}_{\mathrm{k}+1}=\mathrm{C}_{\mathrm{k}}-\mu \mathrm{G}_{\mathrm{k}}
$$

Where $G_{k}$ represents an estimate of the gradient vector, and $C_{k}$ denotes an estimate of the vector of filter coefficients. $\mu$ is a step size $(<<1)$, adjusted to ensure convergence of the iterative routine. 
If $\mathrm{V}_{\mathrm{k}}$ represents the vector of received signal samples, then

and

$$
\mathrm{G}_{\mathrm{k}}=-\mathrm{e}_{\mathrm{k}} \mathrm{V}_{\mathrm{k}}^{*}
$$

$$
\mathrm{C}_{\mathrm{k}+1}=\mathrm{C}_{\mathrm{k}}+\mu \mathrm{e}_{\mathrm{k}} \mathrm{V}_{\mathrm{k}}^{*}
$$

This is the familiar Widrow-Hoff LMS algorithm[6].

The utility of the algorithm is best observed for low $\mathrm{S} / \mathrm{N}$ instances. The $\mathrm{BCM}$ receiver was designed to sense $200 \mathrm{nA}$ of beam current, utilizing a cavity. The typical $\mathrm{S} / \mathrm{N}$ from such a condition is predicted to be $10 \mathrm{~dB}$, so $6 \mathrm{~dB}$ was used for calculations. A FIR was selected with 10 taps, and trial cases presented to the algorithm to quantify any $\mathrm{S} / \mathrm{N}$ improvement. A $\Delta \mathrm{T}$ of $11 \mu \mathrm{s}(20$ samples) and $\mu=0.015$ produced the following results, shown in Figure 4.
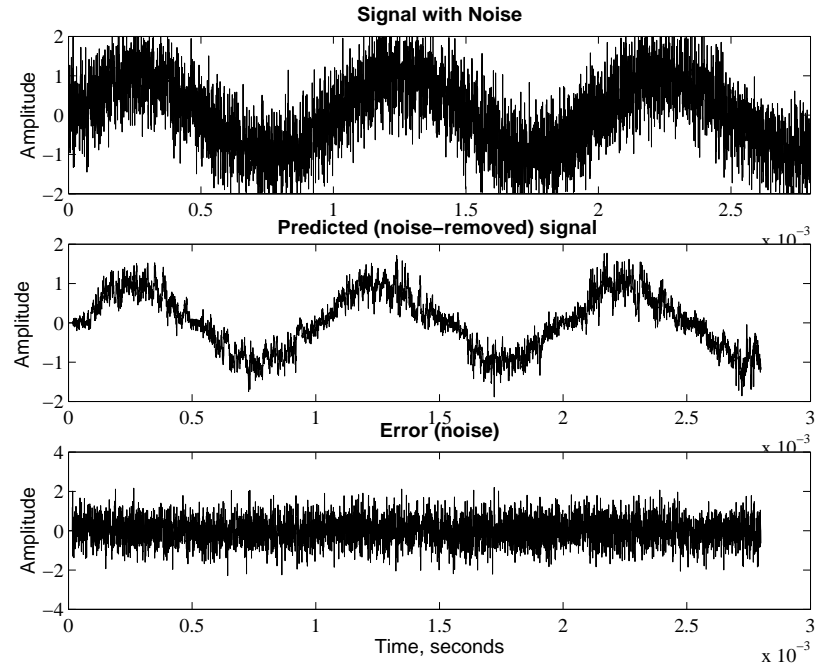

Figure 4: Separation of correlated $(1 \mathrm{kHz})$ signal from uncorrelated noise using LMS algorithm

It is estimated that one iteration of the LMS calculation will take $\sim 100$ clock cycles (at $150 \mathrm{MHz}$ ) to complete[7]. Figure 5 shows the resulting FIR filter response for the 6 $\mathrm{dB}$ S/N case.

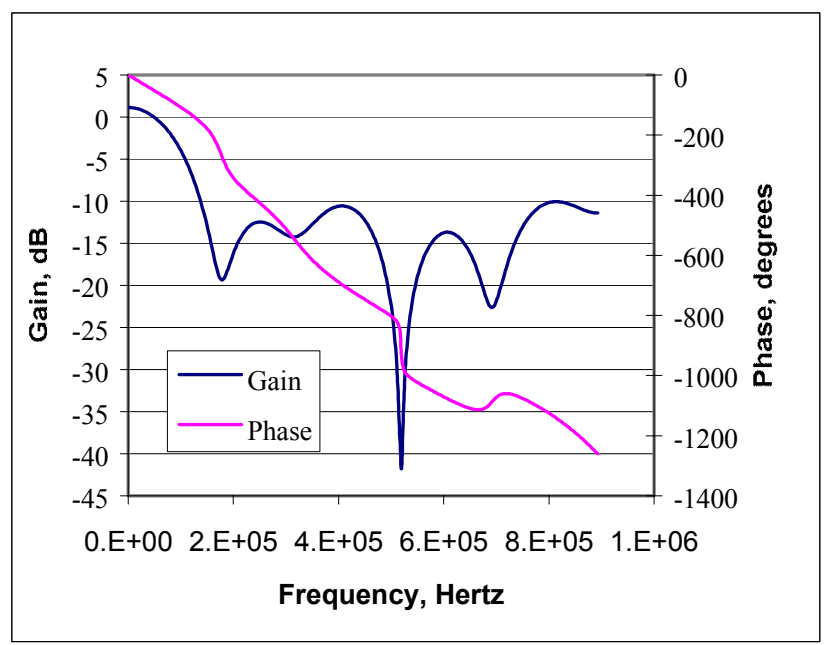

Figure 5:10-tap FIR Bode plot resulting from LMS algorithm; $\mathrm{S} / \mathrm{N}=6 \mathrm{~dB}, \Delta \mathrm{T}=11 \mu \mathrm{s}, \mu=0.015$
It is apparent from the Bode plots that filter magnitude as well as phase response contribute to the filtering mechanism. Most conventional FIR applications concentrate on filter magnitude response, only.

Finally, a "Leaky LMS" design was investigated, to provide only a limited memory, and minimize distortion due to roundoff errors.

\subsection{Adaptive Identifier Configuration}

A variant of the predictor configuration is one of System Identification, shown in Figure 3(b). This is often used to identify the impulse response of a "Black Box" by adjusting the FIR coefficients so as to null the output. The resulting FIR coefficients are then exactly equal to the impulse coefficients of the unknown system.

To use this in a filter application, the unknown system is replaced with an infinite-impulse response (IIR) stage, configured as a single tone oscillator whose controlled parameters are amplitude and phase at a constant frequency $(1 \mathrm{kHz})$. These become the parameters controlled by the LMS algorithm, to minimize the error signal. The resulting IIR amplitude is then used to calculate the signal power. Since received-signal phase is relatively constant, and ultimately not needed, this approach promises to yield the lowest latency, and is currently under investigation.

\section{CONCLUSION}

Digital receiver technology is flexible and configurable and avoids problems associated with non-ideal analog components. In addition, DSP simulation tools are available which model real RF components and imperfect algorithms. This reduces time needed for development, and facilitates faster implementation.

Adaptive filters take advantage of system "knowns" to improve $\mathrm{S} / \mathrm{N}$ ratios by dynamically altering the FIR coefficients. Large $\mathrm{S} / \mathrm{N}$ conditions retain large IF bandwidths, thereby preserving fast transient response. Emphasis is not just placed on producing a filter, but rather a best estimate for the output signal.

\section{REFERENCES}

[1] R. Ursic, et al., "CEBAF Beam Loss Accounting," Proceedings of the 1995 Particle Accelerator Conference, Dallas, TX May, 1995 pp. 2652-4

[2] M. E. Frerking, Digital Signal Processing in Communication Systems, Van Nostrand Reinhold (1994)

[3] MatLab ${ }^{\circledR}$ by The Mathworks, Inc., 24 Prime Park Way, Natick, MA. 01760-1500

[4] System View ${ }^{\circledR}$ by Elanix, Inc., 5655 Lindero Canyon Rd., Suite 721, Westlake Village, CA 91362

[5] Intersil, "HSP50214B PDC Data Sheet," FN 4450-3

[6] J. G. Proakis, Digital Communications, McGraw-Hill, (1989)

[7] S.Kuo, C.Chen "An Implementation of Adaptive Filters with the TMS320C25 or the TMS320C30,"

Texas Instruments, Application Report: SPRA116 1997 